\title{
Bergamot Polyphenols: Pleiotropic Players in the Treatment of Metabolic Syndrome
}

\section{Micaela Gliozzi, Ross Walker and Vincenzo Mollace*}

Institute of Research for Food Safety \& Health (IRC-FSH), University of Catanzaro "Magna Graecia", Catanzaro, Italy

*Corresponding author: Vincenzo Mollace, Campus Universitario di Germaneto, Viale Europa, 88100 Catanzaro, Italy, Tel: 00393274758007; E-mail: mollace@libero.it Received date: March 31, 2014; Accepted date: May 18, 2014; Published date: May 25, 2014

Copyright: ( 2014 Gliozzi M, et al. This is an open-access article distributed under the terms of the Creative Commons Attribution License, which permits unrestricted use, distribution, and reproduction in any medium, provided the original author and source are credited.

\begin{abstract}
Metabolic syndrome (MS) represents a clustering of risk factors related to an elevated incidence of cardiovascular disease (CVD) and type 2 diabetes. Despite the possibility of multiple pharmacological interventions to treat metabolic changes related to MS, these therapeutic strategies often exhibit several side effects and inadequately prevents CVD. Among nutraceutical compounds presenting potential efficacy in this regard, bergamot polyphenols, via their multi-action properties, have been shown to positively modulate several mechanisms involved in MS suggesting their benefits as therapy. The purpose of this review is to discuss the beneficial effects of bergamot polyphenols providing a new therapeutic approach in the treatment of MS.
\end{abstract}

Keywords: Metabolic syndrome; Bergamot polyphenols; Hyperlipidemia; Cardiovascular disease

\section{Introduction}

Metabolic syndrome (MS) is a clustering of numerous age-related metabolic abnormalities that together increase the risk for cardiovascular disease (CVD) and type 2 diabetes. They include obesity which is thought to be a cause rather than a consequence of metabolic disturbance, high blood pressure, high blood glucose and dyslipidaemia [1]. In particular, increased concentrations of lowdensity lipoprotein cholesterol (LDL-C), total blood cholesterol (TC) and triglycerides (TG) comprise the main pathogenic risk profile. Moreover, conditions of insulin resistance such as impaired glucose tolerance or "prediabetes" are often accompanied by low levels of highdensity lipoprotein cholesterol (HDL-C) which amplify the risk of CVD [2].

Recent studies highlight a relationship between dietary factors and MS, but the characteristics of an optimal diet to prevent or treat MS have yet to be better clarified [3]. Increasing experimental and epidemiological evidence suggests that dietary polyphenols, in particular flavonoids, may play an important role in ameliorating prediabetes due to their multi-action properties in counteracting pathophysiological mechanisms leading to the development of MS [4].

The health benefits of polyphenols are generally attributed to both non-specific mechanisms, dependent upon a broad anti-oxidant activity, and more specific mechanisms [5]. Indeed, the in vitro activity of polyphenols strongly suggests that their role extends much beyond their ability to limit oxidative processes as they have been also shown to modulate metabolic enzymes, nuclear receptors, gene expression and multiple signaling pathways [6].

Bergamot (Citrus bergamia) is an endemic plant of the Calabrian region in Southern Italy with a unique profile of flavonoid and flavonoid glycosides present in its juice and albedo, such as neoeriocitrin, neohesperidin, naringin, rutin, neodesmin, rhoifolin and poncirin. Bergamot differs from other Citrus fruits not only because of the composition of its flavonoids, but also because of their particularly high content $[7,8]$. Among them naringin, present also in grapefruit, has already been reported to be active in animal models of atherosclerosis [9], while neoeriocitrin and rutin have been shown to inhibit LDL oxidation [10]. Importantly, bergamot juice is rich in neohesperidosides of hesperetin and naringenin, such as melitidine and brutieridine. These flavonoids possess a 3-hydroxy-3methylglutaryl moiety with a structural similarity to the natural substrate of HMG-CoA reductase and exhibit statin-like proprieties [11].

Recently, the therapeutic potential of bergamot derivatives has also been investigated in human studies [12-14]. Here, we provide a brief overview of these findings underlying the mechanism of action hypothesised for bergamot-derived polyphenols which suggests new and important insights in MS therapy.

\section{Bergamot polyphenolic fraction (BPF) and MS}

The National Cholesterol Education Program Adult Treatment Panel III (NCEPATP III) clinical definition of MS requires the presence of at least three out of five risk factors which include abdominal obesity, high plasma triglycerides, low plasma HDL, high blood pressure and high fasting plasma glucose [15].

Experimental and epidemiological studies have demonstrated that bergamot polyphenolic fraction (BPF) ameliorates serum lipemic profile and normalizes blood pressure in patients suffering from MS. Previous scientific evidence obtained with Citrus flavonoids and other non-nutritive constituents of Citrus fruits, explain their beneficial effects and to further clarify some mechanisms involved in MS $[12,13,16]$.

Indeed, it has been demonstrated that Citrus peel extracts, rich in pectins and flavonoids, cause lowering of cholesterol levels by modulating hepatic HMG-CoA levels $[9,17,18]$ and bergamot juice has been shown to enhance the excretion of fecal sterols in rats [19] thereby contributing to its hypolipemic and hypoglycemic effect subsequently found in patients on BPF treatment. 
A special contribution to the hypolipemic response of BPF seems to be related to the modulatory properties of naringin and neohesperidin. Indeed, evidence exists that dietary hesperetin reduces hepatic TG accumulation and this is associated with the reduced activity of TG synthetic enzymes, such as phosphatidate phosphohydrolase [20]. In addition, in vitro studies suggest that naringenin and hesperein decrease the availability of lipids for assembly of apoB-containing lipoproteins, an effect mediated by reduced activities of acyl CoA: cholesterol acyltransferases (ACAT) [21].

Importantly, BPF is rich in brutieridine and melitidine, which are 3hydroxy-3-methylglutaryl derivatives of hesperetin and naringenin, respectively. In addition, the classical glycoside derivative of naringenin, which is naringin, has been shown to inhibit hepatic HMG-CoA reductase [22]. Therefore it is likely that melitidine and brutieridine in concert with naringin and other flavonone glycosides might be responsible for the striking potency of BPF in reducing cholesterol levels.

The recent finding that eNOS knockout mice present a cluster of cardiovascular risk factors comparable to those of MS suggests that defects in eNOS function may cause human MS and that its dysfunction induces an impaired vasodilation mediated by reduction of NO levels $[15,23,24]$.

The unifying hypothesis of eNOS-reduced activity and subsequent endothelial dysfunction caused by oxidative stress and inflammatory processes observed in MS might justify the reduced NO-dependent vasodilation.

Well documented antioxidant and anti-inflammatory mechanisms regulated by Citrus flavonoids, such as increasing superoxide dismutase and catalase activities and protection of plasma vitamin $\mathrm{E}$ [25], may attenuate overproduction of oxygen reactive species in the vascular wall thereby restoring the imbalanced endothelial function, as also observed in patients under BPF treatment (Figure 1).

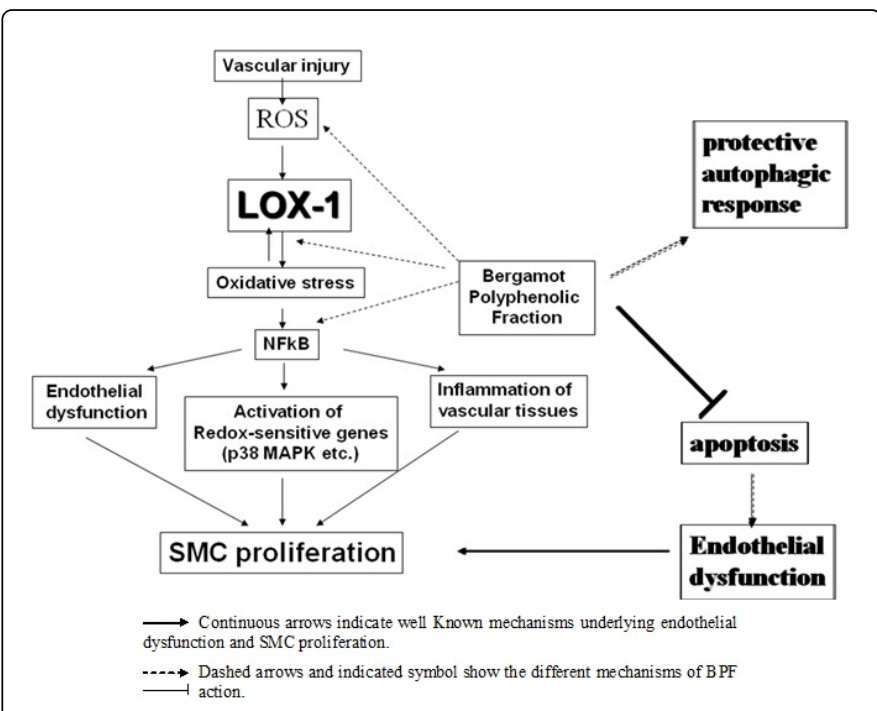

Figure 1: Proposed mechanism of BPF-induced pleiotropic vasoprotective effect

Another potential benefit of BPF is related to its hypoglycemic activity. Among the few mechanistic studies on the hypoglycemic effects of flavonoids, it has been shown that naringenin, similarly to other polyphenols, significantly increased AMP kinase (AMPK) activity and glucose uptake in muscle cells and liver [26,27]. The hypoglycaemic activity of insulin sensitivity and glucose tolerance has been shown in animal models of MS [28].

On the basis of these findings, supplementing an ordinary diet with BPF represents a phytotherapeutic approach for the better management of prediabetic states in patients with MS by lowering plasma cholesterol and lipids, ameliorating NO-dependent vasoreactivity and by reducing blood glucose [12] (Figure 1).

\section{Potential benefits of BPF in reducing statin dosage}

A meta-analysis of placebo-controlled "standard dose" statin trials show a reduction in cardiovascular mortality averaging $20 \%$ and a decrease in major cardiovascular events by approximately $25 \%$ [13]. Treatment with high-dose statins was shown to reduce the morbidity by $36 \%$, and a reduction of cardiovascular events up to $40 \%$ [13]. Despite the significant clinical benefits provided by statins, many patients, in particular those with diabetes or metabolic syndrome do not achieve their recommended LDL-C and HDL-C target goals with statins alone [13]. Moreover, statins have been reported to cause doserelated side effects, the more serious including liver disease or severe myopathy, in up to $22 \%$ of patients eligible for this therapeutic approach [13]. This limits the use of statins and suggests the need for alternative and/or supplementary therapeutic approaches.

The enriched composition of BPF in naringin, neoeriocitrin and neohesperidin produces antilipidemic effects in patients with pure or mixed hypercholesterolemia. This effect is a prominent reduction of both total cholesterol and LDL-C and a moderate increase of HDL-C, thus suggesting a potential benefit in reducing cardiometabolic risk. Given the structural similarity to HMG-CoA reductase substrate brutieridine and melitidine have been shown to possess statin-like properties, by selective inhibition of HMG-CoA reductase [29]. The direct action of BPF on HMG-CoA reductase activity has been confirmed by a significant reduction of the end product of HMG-CoA reductase activity, mevalonate (MVA), detected in the urine of patients under BPF treatment $[12,13,16,30]$.

This effect of BPF suggests a potential benefit of attenuating statininduced side effects through the co-administration of bergamot polyphenols and low dose of statins. Indeed, on the basis of this hypothesis, it has been demonstrated that BPF, given orally in patients with mixed hyperlipidemia, allows the reduction of daily dosage for rosuvastatin but maintain target lipid values of hypolipemic treatment. On the other hand, reduction of serum cholesterol in patients taking both BPF and rosuvastatin is accompanied by a significant reduction in triglyceride levels, an effect which has not been found with rosuvastatin alone, and by a further elevation of HDL-C thus suggesting a synergistic role of BPF in statin-induced hypolipidemic response.

The significant synergism of BPF with rosuvastatin is also demonstrated by the further reduction seen in urinary MVA in patients after treatment with both BPF and lower doses of rosuvastatin [13].

The hypolipidemic response found in patients undergoing BPF treatment seems to be related to the modulatory properties of naringin and neo-hesperidin. Indeed, dietary hesperetin not only reduces the hepatic TG accumulation but also reduces apoB levels [31] which together with an enhanced expression of the LDL receptor may 
explain, at least in part, the hypocholesterolemic properties of BPF. Naringenin shows to act at multiple levels in regulating lipid metabolism in patients [32] probably increasing hepatic fatty acid oxidation through a peroxisome proliferator-activated receptor (PPAR) gamma coactivator alpha/PPARalpha-mediated transcription program, preventing sterol regulatory element-binding protein $1 \mathrm{c}-$ mediated lipogenesis in both liver and muscle by reducing fasting hyperinsulinemia and decreasing hepatic cholesterol and cholesterol ester synthesis. Moreover, naringin is able to reduce both VLDLderived and endogenously synthesized fatty acids, preventing muscle triglyceride accumulation and, finally, improving overall insulin sensitivity and glucose tolerance [33].

In addition to their lipid-lowering properties, BPF synergizes with statins to enhance antioxidant activity. In particular, it has been shown that statins display cholesterol-independent pleiotropic effects including antioxidative actions such as suppression of $\mathrm{NADPH}$ oxidase expression and activity [34,35], induction of antioxidant enzymes (SOD1, SOD3, and GPx) [36,37], prevention of eNOS uncoupling [34,35], and enhancement of eNOS expression and activity. All these beneficial properties are limited by well known side effects of statins; however, this restriction may be overcome by the use of a combination therapy with antioxidants [38]. Indeed, in patients with mixed hyperlipidemia, it has been observed that BPF administration enhances antioxidant properties of rosuvastatin inducing a significant reduction of oxidative stress in circulating polymorphonucleates (PMC). In particular, malonyldialdheyde (MDA) levels, a viable marker of lipid peroxidation, in PMC decreases when adding BPF to rosuvastatin [12,13].

Moreover, the measured oxidative stress in the PMC of patients with hyperlipidemia, treatment with rosuvastatin or BPF alone reduced the expression of LOX-1 and Phospho PKB and these effects were enhanced in patients taking both compounds [13].

Since both LOX-1 and phospho PKB expression are relevant biomarkers of vascular cell viability, it is likely that an additional vasoprotective effect when using both statins and BPF may be expected in patients with high or moderate cardiometabolic risk [13].

\section{Effects of BPF on liver steatosis and LDL particles}

The effect of BPF in lowering cholesterol, triglycerides and glucose in patients suffering from MS is accompanied by reduction of LDL-C and elevation of HDL-C as described above. This beneficial effect in the lipemic profile of patients suffering MS is also characterized by prominent re-arrangement of lipoprotein particle profile found following 120 day BPF treatment. Indeed, BPF reduced small size, atherogenic LDL particles. This effect, combined with reduction of inflammatory biomarkers, suggests that BPF leads to an attenuation of atherogenic risk in patients with MS [14].

The mechanism of such an effect in lipoprotein particle size is not clear to date. The combined effect of BPF in reducing both cholesterol and triglycerides may well explain lipoprotein re-arrangement due to prolonged BPF treatment. Indeed, an increased clearance of TG-rich lipoprotein particles makes these particles became better substrates for lipoprotein lipase. This would be expected to result in decreased levels of large and medium-sized VLDL and perhaps even intermediate density lipoprotein (IDL), which contains roughly equal amounts of TG and cholesterol [14].
The increased cascade of VLDL to IDL to LDL would result in increased numbers of large LDL particles and provide surface constituents for the formation of large HDL. The formation of small LDL is mainly due to cholesteryl ester transfer protein-mediated exchange of VLDL-TG for LDL cholesterol ester and the subsequent hydrolysis of LDL-TG. The decrease in large and medium VLDL diminishes the cholesteryl ester transfer protein-mediated exchange, decreasing the formation and number of small LDL particles. A similar mechanism may also explain the decrease in the number of mediumsized HDL particles.

Recently, it has been shown that MS is associated with non alcoholic fatty liver disease (NAFLD) [14].

The improvement of hepatocyte function found in patients with MS and associated NAFLD after taking BPF might also contribute in the amelioration in lipoprotein profile thereby attenuating cardiometabolic risk [14].

Some studies have demonstrated that insulin resistance almost universally induces NAFLD [39,40]. It is known that this condition may precede the development of cardiovascular disease $[41,42]$. To confirm the connection between NAFLD and atherosclerosis, carotid atherosclerosis has recently been detected in patients with NAFLD [43]. Pathogenetic mechanisms responsible for that include an increased lipolysis and increased delivery of free fatty acids to the liver [44]. The improvement of steato test and hepatorenal index in patients with MS and NAFLD following BPF treatment gives a quantitative estimation of steatosis and leads to the conclusion that BPF improves both liver function and signs of chronic liver inflammation, as confirmed by reduction of TNF- $\triangle$ and CRP [14].

Mild to moderate elevations of serum aminotransferases (ALT and AST) found in BPF-treated patients subjects at baseline represents the most common abnormality found in patients with NAFLD. Their serum levels were significantly reduced after BPF, thereby confirming data obtained with steato test and hepatorenal index.

The mechanism of the hepato-protective effect of BPF still remains to be elucidated. However, evidence shows that BPF acts as a cytoprotective agent in liver of rats administered an high cholesterol diet $[30,45]$. The probable explanation is related to BPF BPF activities in oxidative inflammation and changes in hepatocyte membrane permeability probably via stabilization of the hepatocyte membrane structure, thereby preventing toxins from entering the cells. In addition, other indirect cytoprotective effect may be due to the modulation of hepatic 3-hydroxy-3-methylglutaryl coenzyme A (HMG-CoA) levels, possibly by binding bile acids and increasing the turnover rate of blood and liver cholesterol [46-50], and to the enhancement in the excretion of fecal sterols. As mentioned above, the hypolipidemic response found in patients undergoing BPF treatment may be related to the modulatory properties of naringin and neohesperidin, via inhibition of hepatic TG accumulation. Thus, BPF polyphenolic components, through different mechanisms reduce liver accumulation of fat thereby producing an overall improvement of liver function.

\section{Effects of BPF in a balloon injury model}

It has been demonstrated that bergamot-derived polyphenols are able to antagonize smooth muscle cell (SMC) proliferation and neointima formation in rat carotid artery subsequent to balloon injury. This effect is clearly related to the antioxidant activity of polyphenols, 
as shown by the significant reduction of nitrated tyrosine staining into injured blood vessels, an action due to the reduced generation of peroxynitrite, a powerful oxidant free radical. Moreover, BPF prevented balloon injury-related overexpression of LOX-1, the receptor for oxidized-LDL (oxyLDL), underling the imbalance of redox status of arterial blood vessels [51], thereby leading to SMC proliferation. Peroxynitrite generation is a crucial step in activating proliferation of subintimal SMCs which follows vascular injury; also, LOX-1 expression is involved in this process, which leads to the reactive neointima formation [51].

Oxidative stress and LOX-1 expression are early events in the biochemical changes that can be found in vascular tissue after induction of injury, and restoring antioxidant status by treating rats with BPF reduces restenosis of injured arterial vessels by counteracting free radical formation and LOX-1 expression [16].

In summary, our studies suggest that BPF, a natural antioxidant rich fraction of bergamot (citrus bergamia), inhibits oxidative stress which occurs in injured arteries and modulates both LOX-1 expression and neointima formation. This may be relevant as an alternative approach to conventional anti-atherogenic compounds in the treatment of vascular disorders in which proliferation of vascular SMCs and oxyLDL-related endothelial dysfunction occur [16].

\section{Conclusion}

The nutraceutical approach for the management of MS may represent a promising strategy in preventing cardiometabolic risk. In particular, polyphenols used in clinical practice have been shown to target the pathogenesis of diabetes mellitus, MS and their complications and to favourably modulate a number of biochemical and clinical endpoints [52].

Bergamot-deriving polyphenolic fraction has been shown to possess beneficial effects in patients suffering MS as demonstrated by a concomitant amelioration of lipemic and glycemic profile and by an improvement of the impaired endothelium-mediated vasodilation. In addition, in patients with MS and NAFLD, BPF substantially reduces liver steatosis.

All these effects are due to multi-action properties of bergamot derivatives which modulate key signalling proteins involved in the pathogenesis of MS and, on the other hand, directly counteract oxidative stress shedding new light on the potential use of BPF for reducing cardiometabolic risk in patients with MS.

\section{Acknowledgements}

This paper has been supported by PON a3_00359 and POR Calabria FSE 2007/2013.

\section{References}

1. Cameron A (2010) The metabolic syndrome: validity and utility of clinical definitions for cardiovascular disease and diabetes risk prediction. Maturitas 65: 117-121.

2. Lorenzo C, Wagenknecht LE, D'Agostino RB Jr, Rewers MJ, Karter AJ, et al. (2010) Insulin resistance, beta-cell dysfunction, and conversion to type 2 diabetes in a multiethnic population: the Insulin Resistance Atherosclerosis Study. Diabetes Care 33: 67-72.

3. Cannon CP, Steinberg BA, Murphy SA, Mega JL, Braunwald E (2006) Meta-analysis of cardiovascular outcomes trials comparing intensive versus moderate statin therapy. J Am Coll Cardiol 48: 438-445.
4. Cherniack EP (2011) Polyphenols: planting the seeds of treatment for the metabolic syndrome. Nutrition 27: 617-623.

5. Fraga CG, Galleano M, Verstraeten SV, Oteiza PI (2010) Basic biochemical mechanisms behind the health benefits of polyphenols. Mol Aspects Med 31: 435-445.

6. Seeram NP (2008) Berry fruits: compositional elements, biochemical activities, and the impact of their intake on human health, performance, and disease. J Agric Food Chem 56: 627-629.

7. Dugo P, Presti ML, Ohman M, Fazio A, Dugo G, et al. (2005) Determination of flavonoids in citrus juices by micro-HPLC-ESI/MS. J Sep Sci 28: 1149-1156.

8. Nogata Y, Sakamoto K, Shiratsuchi H, Ishii T, Yano M, et al. (2006) Flavonoid composition of fruit tissues of citrus species. Biosci Biotechnol Biochem 70: 178-192.

9. Jeong YJ, Choi YJ, Choi JS, Kwon HM, Kang SW, et al. (2007) Attenuation of monocyte adhesion and oxidised LDL uptake in luteolin-treated human endothelial cells exposed to oxidised LDL. Br J Nutr 97: 447-457.

10. Yu J, Wang L, Walzem RL, Miller EG, Pike LM, et al. (2005) Antioxidant activity of citrus limonoids, flavonoids, and coumarins. J Agric Food Chem 53: 2009-2014.

11. Di Donna L, De Luca G, Mazzotti F, Napoli A, Salerno R, et al. (2009) Statin-like principles of bergamot fruit (Citrus bergamia): isolation of 3-hydroxymethylglutaryl flavonoid glycosides. J Nat Prod 72: 1352-1354.

12. Mollace V, Sacco I, Janda E, Malara C, Ventrice D, et al. (2011) Hypolipemic and hypoglycaemic activity of bergamot polyphenols: from animal models to human studies. Fitoterapia 82: 309-316.

13. Gliozzi M, Walker R, Muscoli S, Vitale C, Gratteri S, et al. (2013) Bergamot polyphenolic fraction enhances rosuvastatin-induced effect on LDL-cholesterol, LOX-1 expression and protein kinase $B$ phosphorylation in patients with hyperlipidemia. Int J Cardiol 170: 140-145.

14. Gliozzi M, Carresi C, Musolino V, Palma E, Muscoli C et al. (2014) The effect of bergamot-derived polyphenolic fraction on LDL small dense particles and non alcoholic fatty liver disease in patients with MS. Advances in Biological Chemistry.

15. Leighton F, Miranda-Rottmann S, Urquiaga I (2006) A central role of eNOS in the protective effect of wine against metabolic syndrome. Cell Biochem Funct 24: 291-298.

16. Mollace V, Ragusa S, Sacco I, Muscoli C, Sculco F, et al. (2008) The protective effect of bergamot oil extract on lecitine-like oxyLDL receptor-1 expression in balloon injury-related neointima formation. J Cardiovasc Pharmacol Ther 13: 120-129.

17. Nogata Y, Sakamoto K, Shiratsuchi H, Ishii T, Yano M, et al. (2006) Flavonoid composition of fruit tissues of citrus species. Biosci Biotechnol Biochem 70: 178-192.

18. Choe SC, Kim HS, Jeong TS, Bok SH, Park YB (2001) Naringin has an antiatherogenic effect with the inhibition of intercellular adhesion molecule-1 in hypercholesterolemic rabbits. J Cardiovasc Pharmacol 38: 947-955.

19. Vinson JA, Liang X, Proch J, Hontz BA, Dancel J, et al. (2002) Polyphenol antioxidants in citrus juices: in vitro and in vivo studies relevant to heart disease. Adv Exp Med Biol 505: 113-122.

20. Cha JY, Cho YS, Kim I, Anno T, Rahman SM, et al. (2001) Effect of hesperetin, a citrus flavonoid, on the liver triacylglycerol content and phosphatidate phosphohydrolase activity in orotic acid-fed rats. Plant Foods Hum Nutr 56: 349-358.

21. Wilcox LJ, Borradaile NM, de Dreu LE, Huff MW (2001) Secretion of hepatocyte apoB is inhibited by the flavonoids, naringenin and hesperetin, via reduced activity and expression of ACAT2 and MTP. J Lipid Res 42: 725-734.

22. Kim HJ, Oh GT, Park YB, Lee MK, Seo HJ, et al. (2004) Naringin alters the cholesterol biosynthesis and antioxidant enzyme activities in LDL receptorknockout mice under cholesterol fed condition. Life Sci 74: 1621-1634.

23. Mollace V, Muscoli C, Masini E, Cuzzocrea S, Salvemini D (2005) Modulation of prostaglandin biosynthesis by nitric oxide and nitric oxide donors. Pharmacol Rev 57: 217-252.

24. Salvemini D, Kim SF, Mollace V (2013) Reciprocal regulation of the nitric oxide and cyclooxygenase pathway in pathophysiology: relevance and clinical implications. Am J Physiol Regul Integr Comp Physiol 304: R473-487.

25. Jeon SM, Bok SH, Jang MK, Lee MK, Nam KT, et al. (2001) Antioxidative activity of naringin and lovastatin in high cholesterol-fed rabbits. Life Sci 69: 2855-2866. 
26. Hwang JT, Kwon DY, Yoon SH (2009) AMP-activated protein kinase: a potential target for the diseases prevention by natural occurring polyphenols. N Biotechnol 26: $17-22$.

27. Zygmunt K, Faubert B, MacNeil J, Tsiani E (2010) Naringenin, a citrus flavonoid, increases muscle cell glucose uptake via AMPK. Biochem Biophys Res Commun 398: 178-183.

28. Mulvihill EE, Allister EM, Sutherland BG, Telford DE, Sawyez CG, et al. (2009) Naringenin prevents dyslipidemia, apolipoprotein B overproduction, and hyperinsulinemia in LDL receptor-null mice with diet-induced insulin resistance. Diabetes 58: 2198-2210.

29. Gouédard C, Barouki R, Morel Y (2004) Dietary polyphenols increase paraoxonase 1 gene expression by an aryl hydrocarbon receptor-dependent mechanism. Mol Cell Biol 24: 5209-5222.

30. Miceli N, Mondello MR, Monforte MT, Sdrafkakis V, Dugo P, et al. (2007) Hypolipidemic effects of Citrus bergamia Risso et Poiteau juice in rats fed a hypercholesterolemic diet. J Agric Food Chem 55: 10671-10677.

31. Borradaile NM, Carroll KK, Kurowska EM (1999) Regulation of HepG2 cell apolipoprotein B metabolism by the citrus flavanones hesperetin and naringenin. Lipids 34: 591-598.

32. Huong DT, Takahashi Y, Ide T (2006) Activity and mRNA levels of enzymes involved in hepatic fatty acid oxidation in mice fed citrus flavonoids. Nutrition 22: 546-552.

33. Li JM, Che CT, Lau CB, Leung PS, Cheng CH (2006) Inhibition of intestinal and renal Na+-glucose cotransporter by naringenin. Int J Biochem Cell Biol 38: 985-995.

34. Wenzel P, Daiber A, Oelze M, Brandt M, Closs E, et al. (2008) Mechanisms underlying recoupling of eNOS by HMG-CoA reductase inhibition in a rat model of streptozotocin-induced diabetes mellitus. Atherosclerosis 198: 65-76.

35. Antoniades C, Bakogiannis C, Tousoulis D, Reilly S, Zhang MH, et al. (2010) Preoperative atorvastatin treatment in CABG patients rapidly improves vein graft redox state by inhibition of Racl and NADPH-oxidase activity. Circulation 122: S66-73.

36. Carrepeiro MM, Rogero MM, Bertolami MC, Botelho PB, Castro N, et al. (2011) Effect of n-3 fatty acids and statins on oxidative stress in statin-treated hypercholestorelemic and normocholesterolemic women. Atherosclerosis 217 171-178.

37. Landmesser U, Bahlmann F, Mueller M, Spiekermann S, Kirchhoff N, et al. (2005) Simvastatin versus ezetimibe: pleiotropic and lipid-lowering effects on endothelial function in humans. Circulation 111: 2356-2363.

38. Alsheikh-Ali AA, Karas RH (2009) The relationship of statins to rhabdomyolysis malignancy, and hepatic toxicity: evidence from clinical trials. Curr Atheroscler Rep 11: $100-104$.
39. Sanyal AJ; American Gastroenterological Association (2002) AGA technical review on nonalcoholic fatty liver disease. Gastroenterology 123: 1705-1725.

40. Brea A, Mosquera D, Martín E, Arizti A, Cordero JL, et al. (2005) Nonalcoholic fatty liver disease is associated with carotid atherosclerosis: a case-control study. Arterioscler Thromb Vasc Biol 25: 1045-1050.

41. Marchesini G, Brizi M, Morselli-Labate AM, Bianchi G, Bugianesi E, et al. (1999) Association of nonalcoholic fatty liver disease with insulin resistance. Am J Med 107: 450-455

42. Hamaguchi M, Kojima T, Takeda N, Nagata C, Takeda J, et al. (2007) Nonalcoholic fatty liver disease is a novel predictor of cardiovascular disease. World J Gastroenterol 13: 1579-1584.

43. Chitturi S, Abeygunasekera S, Farrell GC, Holmes-Walker J, Hui JM, et al. (2002) NASH and insulin resistance: Insulin hypersecretion and specific association with the insulin resistance syndrome. Hepatology 35: 373-379.

44. Choudhury J, Sanyal AJ (2004) Insulin resistance and the pathogenesis of nonalcoholic fatty liver disease. Clin Liver Dis 8: 575-594, ix.

45. Janda E, Parafati M, Aprigliano S, Carresi C, Visalli V, et al. (2013) The antidote effect of quinone oxidoreductase 2 inhibitor against paraquat-induced toxicity in vitro and in vivo. Br J Pharmacol 168: 46-59.

46. Bok SH, Lee SH, Park YB, Bae KH, Son KH, et al. (1999) Plasma and hepatic cholesterol and hepatic activities of 3-hydroxy-3-methyl-glutaryl-CoA reductase and acyl CoA: cholesterol transferase are lower in rats fed citrus peel extract or a mixture of citrus bioflavonoids. J Nutr 129: 1182-1185.

47. Marounek M, Volek Z, Synytsya A, Copíková J (2007) Effect of pectin and amidated pectin on cholesterol homeostasis and cecal metabolism in rats fed a highcholesterol diet. Physiol Res 56: 433-442.

48. Terpstra AH, Lapre JA, de Vries HT, Beynen AC (1998) Dietary pectin with high viscosity lowers plasma and liver cholesterol concentration and plasma cholesteryl ester transfer protein activity in hamsters. J Nutr 128: 1944-1949.

49. Terpstra AH, Lapré JA, de Vries HT, Beynen AC (2002) The hypocholesterolemic effect of lemon peels, lemon pectin, and the waste stream material of lemon peels in hybrid F1B hamsters. Eur J Nutr 41: 19-26.

50. Garcia-Diez F, Garcia-Mediavilla V, Bayon JE, Gonzalez-Gallego J (1996) Pectin feeding influences fecal bile acid excretion, hepatic bile acid and cholesterol synthesis and serum cholesterol in rats. J Nutr 126: 1766-1771.

51. Muscoli C, Sacco I, Alecce W, Palma E, Nisticò R, et al. (2004) The protective effect of superoxide dismutase mimetic M40401 on balloon injury-related neointima formation: role of the lectin-like oxidized low-density lipoprotein receptor-1. J Pharmacol Exp Ther 311: 44-50.

52. Davì G, Santilli F, Patrono C (2010) Nutraceuticals in diabetes and metabolic syndrome. Cardiovasc Ther 28: 216-226. 